\title{
Fluctuation properties of strength functions associated with giant resonances
}

\author{
Hirokazu Aiba, ${ }^{1}$ Masayuki Matsuo, ${ }^{2}$ Shigeru Nishizaki, ${ }^{3}$ and Toru Suzuki ${ }^{4}$ \\ ${ }^{1}$ Kyoto Koka Women's College, 38 Kadono-cho Nishikyogoku, Ukyo-ku, 615-0882 Kyoto, Japan \\ ${ }^{2}$ Graduate School of Science and Technology, Niigata University, 950-2181 Niigata, Japan \\ ${ }^{3}$ Faculty of Humanities and Social Sciences, Iwate University, 3-18-34 Ueda, 020-8550 Morioka, Japan \\ ${ }^{4}$ Department of Physics, Tokyo Metropolitan University, 192-0397 Hachioji, Japan
}

(Received 28 May 2003; published 24 November 2003)

\begin{abstract}
We performed fluctuation analysis by means of the local scaling dimension for the strength function of the isoscalar (IS) and the isovector (IV) giant quadrupole resonances in ${ }^{40} \mathrm{Ca}$, where the strength functions are obtained by the shell model calculation within up to the $2 p 2 h$ configurations. It is found that at small energy scale, fluctuation of the strength function almost obeys the Gaussian orthogonal ensemble (GOE) random matrix theory limit. On the other hand, we found a deviation from the GOE limit at the intermediate energy scale of about $1.7 \mathrm{MeV}$ for the IS and at $0.9 \mathrm{MeV}$ for the IV. The results imply that different types of fluctuations coexist at different energy scales. Detailed analysis strongly suggests that GOE fluctuation at small energy scale is due to the complicated nature of $2 p 2 h$ states and that fluctuation at the intermediate energy scale is associated with the spreading width of the Tamm-Dancoff $1 p 1 h$ states.
\end{abstract}

DOI: 10.1103/PhysRevC.68.054316

PACS number(s): 24.60.Ky, 21.10.-k, 24.30.Cz

\section{INTRODUCTION}

Strengths of exciting high lying states in nuclei (for instance, a giant resonance) are spread over a certain energy interval due to the coupling to a background which is complicated and has huge degrees of freedom. As a result, the strength function, at a large energy scale, exhibits a global shape profile like a Lorentzian. The values of the center of energy or the total width depend on the properties of a probe such as multipolarlity, isospin, and so on, and change smoothly as the mass number of a nucleus. Most of these values are well understood up to now [1-3]. On the other hand, at small energy scale limit comparable to the level spacing of the background states, the strength function may rapidly fluctuate from state to state on top of the global profile, if we neglect the escaping width due to the coupling to the continuum. It is believed that the fluctuation properties at this energy scale can be well simulated by the random matrix theory of the Gaussian orthogonal ensemble (GOE) [4-7], which has been verified by several experimental data: The nearest-neighbor level spacing distribution (NND) of neutron resonances near the threshold approximately follows the Wigner distribution $[7,8]$. The distribution of the reduced width (proportional to an absolute square of a component of the wave function) of these resonances also shows the Porter-Thomas distribution [7,8]. Furthermore, the NND of the nuclear data ensemble shows the Wigner distribution and the $\Delta_{3}$ statistics of them shows $\Delta_{3}(L) \propto \ln L$ [9]. All of these are typical signatures of the GOE.

Thus, we know well the behavior of the strength function at both extremely small and large energy scales. From this knowledge, one may construct the following model to describe the strength function of highly excited states in nuclei: A collective state couples democratically to each of unperturbed background states, and the background Hamiltonian itself is the GOE random matrix. This GOE background model is closely connected with the pandemonium picture
$[10,11]$. If we adjust the energy of the collective state and the coupling strength between the collective state and background states, the resultant strength function should be consistent with the giant resonance strength function at least at both extreme energy scale limits. Therefore, in order to find the difference between a realistic strength function and that from this model, we must investigate fluctuation properties of the strength function at intermediate energy scale $[12,13]$.

Recently, we proposed a new method to perform fluctuation analysis of the strength function [14]. This method is devised to quantitatively characterize a fluctuation property as a function of energy scale by a new measure called the local scaling dimension (LSD), and is suitable to investigate fluctuation at intermediate energy scales. We applied this method to a simple doorway damping model. The doorway damping model is different from the above GOE background model with respect to two points. First, unperturbed background states are divided into two different classes so that unperturbed states in one class, which are called the doorway states, couple directly to a collective state, while states in another class do not. The second point is that doorway states and other unperturbed background states have a finite spreading width due to the mixing among them. (Note that the GOE background model corresponds to the large spreading width limit.) Fluctuation properties of the strength functions of both models are same at small energy scale limit. The result of the analysis clearly showed that fluctuation properties of both strength functions deviate from each other at a certain intermediate energy scale, and this energy scale is closely related to the spreading width of the doorway states in the doorway damping model.

In this paper, we apply the same method to a more realistic strength function of the giant resonances in a nucleus. Strength functions of the isoscalar (IS) and the isovector (IV) giant quadrupole resonance $(\mathrm{GQR})$ in ${ }^{40} \mathrm{Ca}$ are obtained by the shell model calculation within up to the $2 p 2 h$ configurations. The strength functions reproduce the experimental cen- 

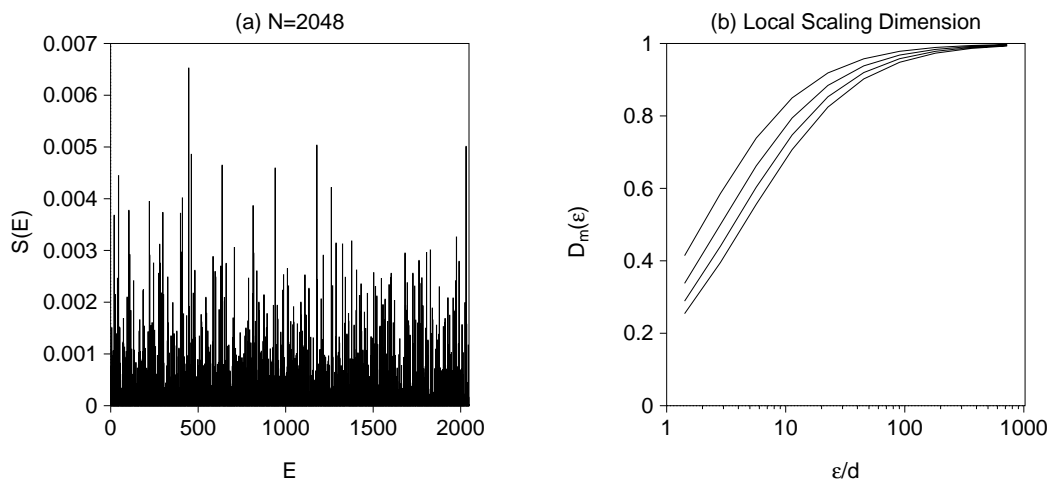

FIG. 1. (a) An example of the GOE strength function with matrix dimension of 2048. (b) The local scaling dimension $D_{m}(\epsilon)$ for the GOE strength function obtained after the ensemble average. The curves correspond to $D_{m}(\epsilon)$ for $m$ $=2-5$ from upper to lower.

ter of energy and the total width, and also show the GOE fluctuation at the small energy scale limit, as will be shown later. Furthermore, in this model, the doorway structure and the deviation from GOE in the background may be introduced in a natural way, while they are input by hand in the doorway damping model. Therefore these strength functions may provide the better test for the analysis.

Note that a similar method of analysis was proposed and applied to the $\left(e, e^{\prime}\right)$ or $\left(p, p^{\prime}\right)$ experimental data on ${ }^{208} \mathrm{~Pb}$ $[15,16]$.

The paper is organized as follows: In Sec. II, we briefly explain the LSD. In Sec. III, we apply the method to the strength functions of the IS and the IV GQR. We also discuss the physical origin of the deviation from the GOE background model in detail. Finally, Sec. IV is devoted to conclusions.

\section{LOCAL SCALING DIMENSION}

We briefly explain the local scaling dimension in this section. See Ref. [14] for detail.

The strength function is expressed [17] as

$$
S(E)=\sum_{i} S_{i} \delta\left(E-E_{i}+E_{0}\right)
$$

for exciting the nucleus with excitation energy $E$. Here $E_{i}$ and $E_{0}$ are the energy of discrete levels and the ground state energy, respectively, and $S_{i}$ denotes the strength of exciting the $i$ th energy level. Let us assume that strengths are normalized as $\Sigma_{i} S_{i}=1$.

In order to quantitatively characterize how the strength function fluctuates at various energy scale, we consider binned distribution of the strength function $S(E)$ by dividing whole energy interval under consideration into $L$ bins with length $\epsilon$. Strength contained in $n$th bin is denoted by $p_{n}$,

$$
p_{n} \equiv \sum_{i \in n \text {th bin }} S_{i}
$$

To characterize the distribution of the binned strengths, we introduce the moments of it, which are called, in literature, the partition function $\chi_{m}(\epsilon)$ defined by

$$
\chi_{m}(\boldsymbol{\epsilon}) \equiv \sum_{n=1}^{L} p_{n}^{m}=L\left\langle p_{n}^{m}\right\rangle .
$$

Clearly, the partition functions contain, at small $\epsilon$ limit, the information of the strength distribution, as well as the energy-strength correlation as a function of a bin width or an energy scale $\epsilon$.

It is then helpful to employ the idea of the generalized fractal dimension $[18,19]$, which is derived from the partition function and has a definite physical meaning. The concept of the generalized fractal dimension is useful only when a system has a multifractal nature, namely, has a self-similar structure against the change of the scale. Strength functions at a nucleus generally do not have such a property. Thus, we have to extend the concept of the generalized fractal dimension and finally reach the LSD defined by

$$
D_{m}(\epsilon) \equiv \frac{1}{m-1} \frac{\partial \log \chi_{m}(\epsilon)}{\partial \log \epsilon} .
$$

Different from the generalized fractal dimension, the local scaling dimension characterizes "how the partition func-
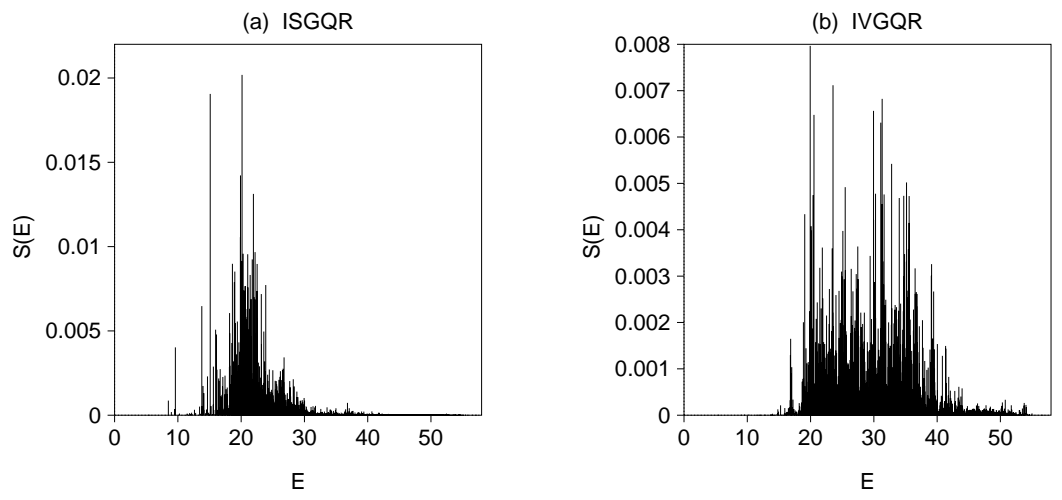

FIG. 2. Strength functions for ISGQR (a) and IVGQR (b). 
tion scales for different energy scales" and accordingly is a function of a bin width or an energy scale $\epsilon$. The local scaling dimension, however, has an easily understandable physical meaning similar to those of the generalized fractal dimension. For instance, the value of $D_{m}(\epsilon)$ close to unity means that the strength function looks like a onedimensional object distributed smoothly over an energy interval when we look at the strength function at an energy scale $\epsilon$. On the other hand, it looks like a zerodimensional object, namely, a dot if the value of $D_{m}(\epsilon)$ is close to zero. Note that the local scaling dimension reduces to the generalized fractal dimension when the value of $D_{m}(\epsilon)$ is constant over a whole interval of $\epsilon$.

In actual calculation of the local scaling dimension, we define it by means of the finite difference under the change of a factor 2 ,

$$
D_{m}(\sqrt{2} \epsilon)=\frac{1}{m-1} \frac{\log \chi_{m}(2 \epsilon)-\log \chi_{m}(\epsilon)}{\log 2},
$$

rather than the derivative in Eq. (4). Using the finite difference, the calculation is very simple for all the moments.

One of the important strength functions may be the one obtained from the GOE random matrix. The local scaling dimension of this GOE strength function can be used as a reference to study other strength functions which are supposed to have fluctuation properties similar to the GOE, as in the case of nuclear strength functions in the highly excited region. We show in Fig. 1 an example of the GOE strength function and the local scaling dimension $D_{m}(l)$, where $l$ $\equiv \epsilon / d$ (the bin width measured in the unit of the level spacing $d$ ) is used as the scaling parameter.

\section{ANALYSIS OF THE GIANT QUADRUPOLE RESONANCES IN ${ }^{40} \mathrm{Ca}$}

We apply the local scaling dimension to the strength functions of the giant quadrupole resonances in ${ }^{40} \mathrm{Ca}$.

\section{A. Numerical calculation of strength functions}

We calculated the strength functions of the isoscalar and the isovector giant quadrupole resonances in ${ }^{40} \mathrm{Ca}$ within the second Tamm-Dancoff approximation including the $1 p 1 h$ and $2 p 2 h$ excitations. Single-particle wave-functions and energies were obtained in terms of a Woods-Saxon potential including the Coulomb interaction. To simulate the bare (Hartree-Fock) single-particle energies $\varepsilon_{\mathrm{HF}}$, the above singleparticle energies $\varepsilon_{\mathrm{WS}}$ were scaled by the effective mass $\mathrm{m}^{*} / \mathrm{m}$ as $\varepsilon_{\mathrm{HF}}=\varepsilon_{\mathrm{WS}}\left(\mathrm{m}^{*} / \mathrm{m}\right)$. As the residual interaction, the LandauMigdal-type interaction [20] including the densitydependence was adopted. The model space was constructed in terms of single-particle states within the four major shells, two below and two above the Fermi surface, and included all $1 p 1 h$ and $2 p 2 h$ states whose unperturbed energies were less than $50 \mathrm{MeV}$. Resultant number of $1 p 1 h$ states and $2 p 2 h$ states are 34 and 4144, respectively. We diagonalized the

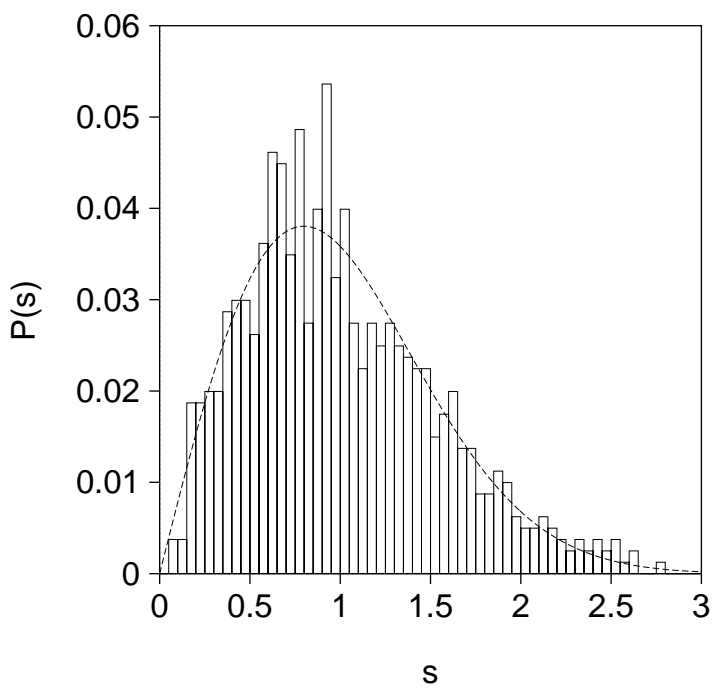

FIG. 3. The nearest-neighbor level spacing distribution for levels between 20 and $30 \mathrm{MeV}$. Level spacings were unfolded by the Strutinsky method with a smoothing width of $5 \mathrm{MeV}$. The dashed curve represents the Wigner distribution.

Hamiltonian within this model space and obtained the strength functions for the isoscalar and the isovector quadrupole operators.

Figures 2(a) and 2(b) show the strength functions for ISGQR and IVGQR, respectively. The mean energy and the total width (the standard deviation) are $23 \mathrm{MeV}(31 \mathrm{MeV})$ and $4.0 \mathrm{MeV}(7.0 \mathrm{MeV})$ for the ISGQR (IVGQR). The effective mass $\mathrm{m}^{*} / \mathrm{m}=0.9$ was used to reproduce the empirical mean energy for IVGQR in ${ }^{40} \mathrm{Ca}$ [3]. These strength functions were already used in Refs. [21] and [22] to study the generic properties of nuclear giant-resonance decay.

\section{B. Several measures}

Before going to the detailed discussion for the local scaling dimension, we briefly show other fluctuation measures.

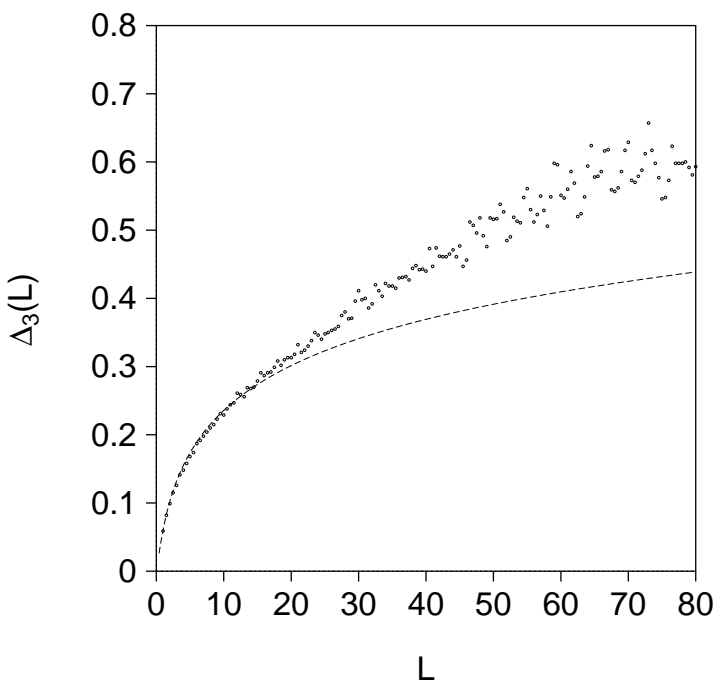

FIG. 4. The $\Delta_{3}$ statistics. The dashed curve represents the $\Delta_{3}$ for the GOE level fluctuation. Same as Fig. 3 for others. 

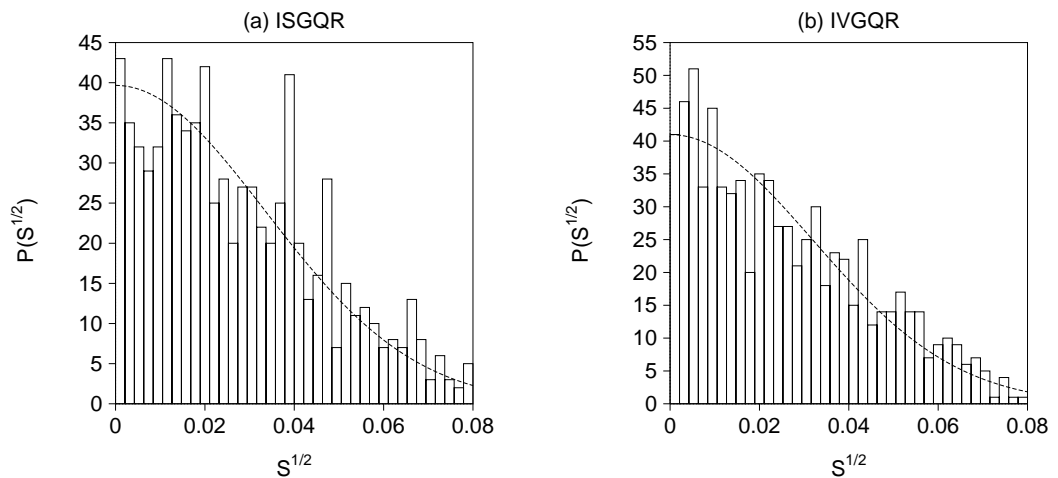

FIG. 5. The statistical distribution of square root of unfolded strengths $\bar{S}_{i}^{1 / 2}$, associated with the individual levels between 20 and $30 \mathrm{MeV}$ for (a) ISGQR and (b) IVGQR. The dashed curve represents the Porter-Thomas distribution which becomes a Gaussian when plotted as a function of $\bar{S}_{i}^{1 / 2}$.

Figure 3 shows the NND, where levels between $20 \mathrm{MeV}$ and $30 \mathrm{MeV}$ are considered (the number of levels is 804), and are unfolded by means of the Strutinsky method with a smoothing width of $5 \mathrm{MeV}$ [23]. The NND follows the Wigner distribution rather well, which indicates that at the very small energy scale the level fluctuation almost obeys the GOE. The importance of the particle-hole type residual interaction among $2 p 2 h$ states to generate GOE level fluctuation was discussed in Ref. [24]. We also plot in Fig. 4 the $\Delta_{3}$ statistics. We again find that at small energy range the $\Delta_{3}$ follows the GOE line. At $L^{\max } \simeq 15$, however, $\Delta_{3}$ starts to deviate from the GOE line to upward. This value of $L^{\max }$ roughly corresponds to the energy scale $E \simeq 0.18 \mathrm{MeV}$.

Figure 5 shows the strength distribution where the distribution of the square root of normalized strengths $\bar{S}_{i}$ is plotted as a histogram; strengths are normalized also by Strutinsky method, detailed procedure of which will be explained in the following section. We can see that the strength distribution follows considerably well the Porter-Thomas distribution.

\section{Results of the local scaling dimension}

Since we are not interested in the global shape of the strength function, it is convenient to introduce the normalized strength function where the global smooth energy dependence is removed from the original strength function as follows:

$$
\bar{S}_{i}=\mathcal{N} \frac{S_{i} \widetilde{\rho}\left(E_{i}\right)}{\widetilde{S}\left(E_{i}\right)} .
$$

Here, $\tilde{S}(E)$ and $\widetilde{\rho}(E)$ are obtained by averaging the calculated strength function [Eq. (1)] and the level density
$\rho(E)=\Sigma_{i} \delta\left(E-E_{i}+E_{0}\right)$, respectively, by the Strutinsky method. We adopted $5 \mathrm{MeV}$ as a smoothing width. $\mathcal{N}$ is an overall normalization factor to guarantee $\Sigma_{i} \bar{S}_{i}=1$ for the considered energy interval. Moreover, we adopt as energy levels the equidistant ones [14], namely, $E_{i}=i d$, where $d$ denotes average level spacing. By adopting these levels, we can neglect global energy dependence of the level density and the local level fluctuation. (We verified that the inclusion of the local level fluctuation affects only the behavior of the LSD at small energy scales, and this effect can be described by the GOE.) Thus, we obtain the normalized strength function $\bar{S}(E)$;

$$
\bar{S}(E)=\Sigma_{i} \bar{S}_{i} \delta\left(E-E_{i}+E_{0}\right) .
$$

The local scaling dimension is derived from this normalized strength function. Finally, since the visible strengths of ISGQR are almost concentrated in the energy range between $20 \mathrm{MeV}$ and $30 \mathrm{MeV}$, we adopt this energy interval for analysis. The normalized strength functions for ISGQR and IVGQR are plotted in Figs. 6(a) and 6(b), respectively.

First, let us discuss the ISGQR case in detail. The case of the IVGQR will be discussed in Sec. III D 4. Figures 7(a) and $7(\mathrm{~b})$ show the partition functions $\chi_{m}(\epsilon)$ defined by Eq. (3) and the local scaling dimensions $D_{m}(\epsilon)$ defined by Eq. (5), respectively. The horizontal axes in both figures represent the bin width of energy axis in unit of $d$, where $d$ represents the average level spacing over the energy range $20-30 \mathrm{MeV}(d \simeq 12 \mathrm{keV})$. From the partition function $\chi_{m}(\epsilon)$, one sees that the fluctuation does not follow the power scaling law (or linear relation in the log-log plot). Correspondingly, the local scaling dimension $D_{m}(\epsilon)$ varies as a function
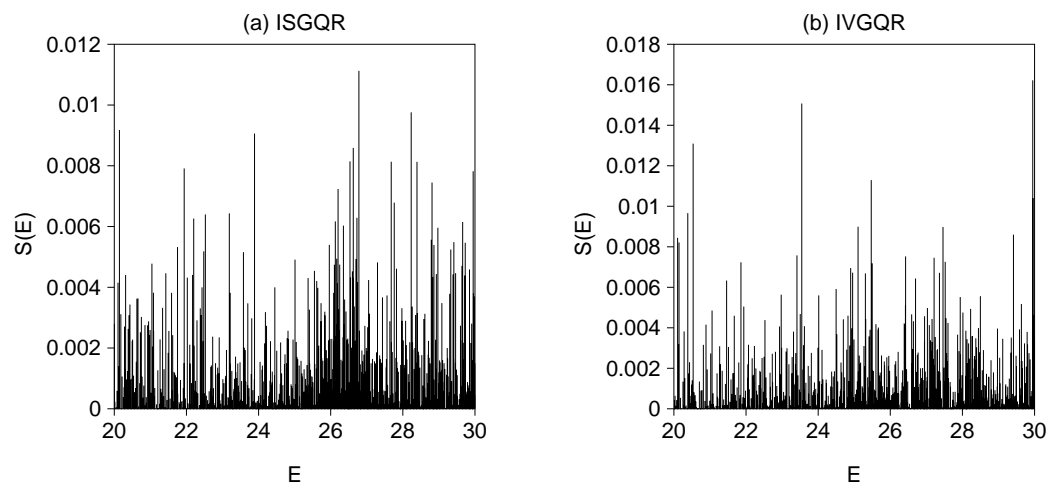

FIG. 6. The normalized strength functions $\bar{S}(E)$ corresponding to the original ones for the ISGQR (a) and for the IVGQR (b) plotted in Fig. 2. A summation of strengths within an energy interval $20-30 \mathrm{MeV}$ is normalized to unity. 

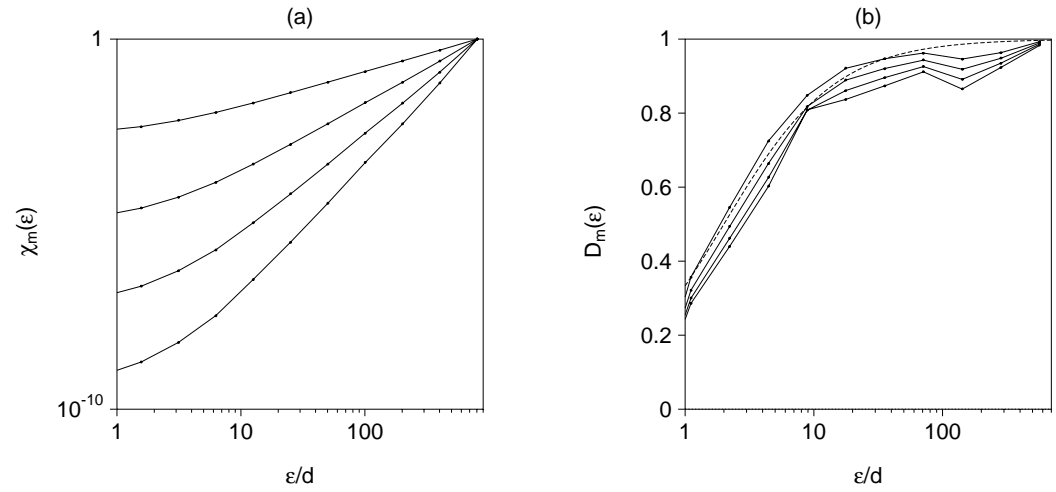

FIG. 7. (a) The partition function $\chi_{m}(\epsilon)$ for $m=2-5$ for the ISGQR corresponding to the normalized strength function plotted in Fig. 6. (b) Its local scaling dimension $D_{m}(\epsilon)$ for $m=2-5$. The dashed curve represents $D_{2}(\epsilon)$ for the GOE.

of $\epsilon$. At small energy scale, the values of $D_{m}(\epsilon)$ gradually increase as the energy scale $\epsilon$ increases, and almost follow the GOE line. As the energy scale $\epsilon$ increases further, the values of $D_{m}(\epsilon)$ bend downwards and then again turn to increase. The behavior of $D_{m}(\epsilon)$ around this energy scale clearly deviates from the GOE limit. The value of the energy scale where the deviation is maximum is roughly $\epsilon_{\mathrm{f}} \simeq 140 \mathrm{~d}$ $\simeq 1.7 \mathrm{MeV}$. From these observations, we again find that at small energy scale, fluctuation of the strength function is essentially governed by the GOE, as one knows from other measures such as the NND or the strength distribution. The scaling analysis by means of the local scaling dimension, however, can reveal the new feature which may not be realized by other measures. Namely, the GOE fluctuation is limited to the small energy scale, while, at intermediate energy scales, a different fluctuation from the GOE arises.

One may wonder that the statistical error could produce a fictitious dip, since the number of levels considered 804 is not so large. Of course, there may be a statistical error. Accordingly, we should take the energy scale $\epsilon_{\mathrm{f}} \simeq 140 d$ $\simeq 1.7 \mathrm{MeV}$ mentioned above as a rough estimate. The existence of the dip itself, however, is not due to a statistical error. This would be verified from a systematic analysis in the following section. Note that the simple estimate of the standard deviation of the $m$ th local scaling dimension is $\sigma\left(D_{m}\right) \simeq \sqrt{3} m / \sqrt{N_{\text {tot }}}[14]$, and in the present case for the second local scaling dimension, $\sigma\left(D_{2}\right) \simeq 0.12$.

A type of the smoothing procedure to obtain the normalized strength function does not much affect the results. Indeed, we verified that the Gaussian smoothing instead of the Strutinsky smoothing leads to the essentially same results.

\section{Origin of the deviation from the GOE}

We shall investigate the origin of the observed energy scale $\epsilon_{\mathrm{f}}=1.7 \mathrm{MeV}$ characteristic to the fine structures in the isoscalar quadrupole strength function.

\section{Doorway damping mechanism}

For this purpose we consider a damping mechanism by separating the shell model space to $1 p 1 h$ and $2 p 2 h$ subspaces, and we focus on the residual interactions within and between the subspaces. The giant resonance is a collective vibrational state whose dominant component is a coherent superposition of $1 p 1 h$ configurations. The collective state can be described by the Tamm-Dancoff (TD) approximation, which corresponds to a truncated shell model where only $1 p 1 h$ configurations are taken into account. The result of the TD approximation (TDA) is shown in Fig. 8(a), and is compared with the full shell model calculation including up to $2 p 2 h$ 's [Fig. 2(a)]. It is seen that the peak position is well reproduced by the TDA. Note also that the isoscalar quadrupole strength in the TDA is spread over many eigenstates, which are correlated $1 p 1 h$ states. This spreading of the quadrupole strength among $1 p 1 h$ states is often called the Landau damping. Comparing Figs. 8(a) and 2(a), the Landau damping is found to give dominant contribution to the total full width at half maximum (FWHM) of the strength function.

If we take into account the $2 p 2 h$ configurations, the interaction acting between $1 p 1 h$ and $2 p 2 h$ states (abbreviated as $V_{12}$ ) takes part. Since the $2 p 2 h$ states have much more degrees of freedom, $V_{12}$ causes spreading of the TD $1 p 1 h$ states. Because of this spreading of $1 p 1 h$ states, the quadru-
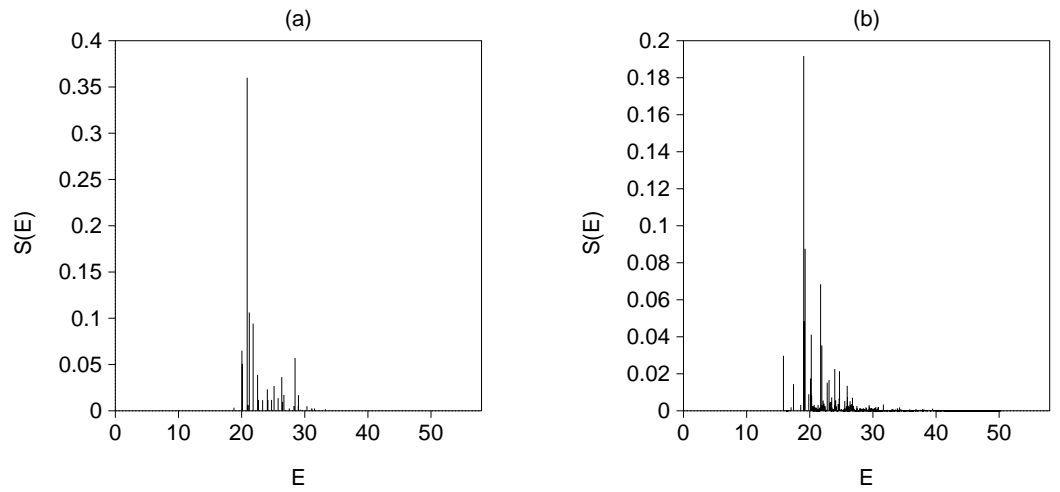

FIG. 8. (a) The strength function for the ISGQR obtained within the Tamm-Dancoff approximation and (b) the strength function for the ISGQR by omitting the coupling among the unperturbed $2 p 2 h$ states. 


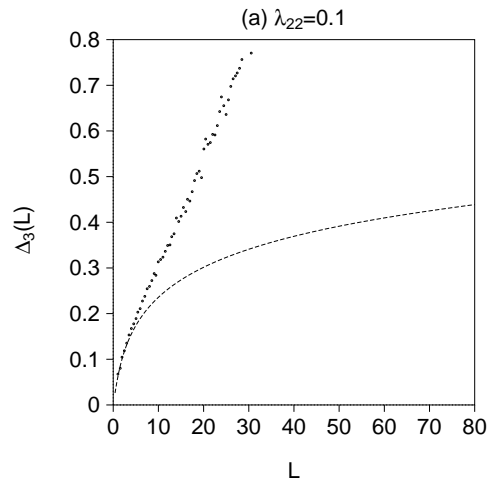

pole strength is also fragmented by $V_{12}$ on top of the Landau damping at the TDA level. To see the effect of $V_{12}$, we performed a calculation where unperturbed $2 p 2 h$ states and the interaction $V_{12}$ is introduced. The resultant strength distribution, shown in Fig. 8(b), appears similar to the final result [Fig. 2(a)] as far as the gross profile of the strength distribution is concerned.

Note also that there exists the residual interaction acting among $2 p 2 h$ states, which we call $V_{22}$ in the following. $V_{22}$ causes configuration mixing among the $2 p 2 h$ states. Comparison between Figs. 8(b) and 2(a) indicates effects of $V_{22}$ on fine details of strength distribution, although the gross profile of strength distribution is not influenced very much.

The above observations lead us to the following doorway damping picture. The quadrupole strength distribution is fragmented first in the $1 p 1 h$ subspace. The $1 p 1 h$ TD states then spread further over $2 p 2 h$ states through $V_{12}$. Here the $1 p 1 h$ TD states may be considered as doorway states in the whole damping processes. The $2 p 2 h$ states on the other hand may be regarded as states that play roles of background in the main damping processes since they do not dominate the total damping width, but they influence the fine structures through the coupling to the doorway states.

Assuming the above picture, we can consider energy scales that may be relevant to the fine structures of the quadrupole strength distribution. Concerning the $1 p 1 h$ TD states which are considered as the doorway states, we have as energy scales (1) the spreading width (which we denote $\gamma_{12}$ ) of the TD $1 p 1 h$ states caused by the interaction $V_{12}$, in addition to (2) the average level spacing $d_{1 p 1 h}$ between the $1 p 1 h$ states. Concerning the $2 p 2 h$ states, (3) the level spacing $d_{2 p 2 h}$ between $2 p 2 h$ states is to be noted. $\left(d_{2 p 2 h}\right.$ is almost identical to the level spacing $d$ for the whole set of energy spectra since the number of $2 p 2 h$ states is much larger than that of $1 p 1 h$ states.) Note also that mixing among $2 p 2 h$ states caused by the interaction $V_{22}$ is characterized by (4) the spreading width ( $\gamma_{22}$ named in the following) of $2 p 2 h$ states. We have found previously that, in the case of a schematic model which incorporates doorway states coupled to background states, the spreading width of doorway states (corresponding to $\gamma_{12}$ in the present context) determines the energy scale where the local scaling dimension deviates from the generic GOE limit [14]. Note also that the deviation from the GOE is seen in the energy level fluctuation by the analysis of the $\Delta_{3}$ statistics. The energy level fluctuation is sensitive to the residual interaction that causes mixing among unper-
FIG. 9. The $\Delta_{3}$ statistics with rescaling the interaction strength $V_{22}$ by a factor $\lambda_{22}$, for (a) $\lambda_{22}=0.1$ and (b) $\lambda_{22}=2.0$. The dashed curve represents the $\Delta_{3}$ for the GOE level fluctuation. We can find the large dependence of the $\Delta_{3}$ on the value of $V_{22}$. We also verified that the $\Delta_{3}$ is insensitive to the change of the value of $V_{12}$, namely, the behavior of $\Delta_{3}$ at $\lambda_{12}=0.1$ or $\lambda_{12}$ $=2.0$ is almost same as that at $\lambda_{12}=1.0$, where $\lambda_{12}$ represents the rescaling factor of the interaction strength $V_{12}$. turbed configurations. We found that the energy level fluctuation is governed by $V_{22}$, but not by $V_{12}$, as shown in Fig. 9. Thus we can consider another possibility that the energy scale of the deviation from the GOE limit may be related to the spreading width $\gamma_{22}$ of the $2 p 2 h$ states. In the following, we shall focus on the effects of $V_{12}$ and $V_{22}$ which are connected to the two spreading widths $\gamma_{12}$ and $\gamma_{22}$.

\section{Spreading widths of $1 p 1 h$ and $2 p 2 h$ states}

Let us quantify $\gamma_{12}$ and $\gamma_{22}$. If we assume the Fermi golden rule, we obtain a simple estimate of $\gamma_{12}$ by $\gamma_{12}^{\mathrm{FG}}$ $=2 \pi \overline{\left\langle 1 p 1 h\left|V_{12}\right| 2 p 2 h\right\rangle^{2}} / d_{2 p 2 h}$, where $\overline{\left\langle 1 p 1 h\left|V_{12}\right| 2 p 2 h\right\rangle}$ is a root mean square value of the matrix elements between $1 p 1 h$ and $2 p 2 h$ states. Similarly, we can make an estimation of the spreading width $\gamma_{22}$ as $\gamma_{22}^{\mathrm{FG}}=2 \pi \overline{\left\langle 2 p 2 h\left|V_{22}\right| 2 p 2 h\right\rangle^{2}} / d_{2 p 2 h}$. The above estimate gives $\gamma_{12}^{\mathrm{FG}} \simeq 5.3 \mathrm{MeV}$ and $\gamma_{22}^{\mathrm{FG}} \simeq 6.0 \mathrm{MeV}$. Here we have inserted $\overline{\left\langle 1 p 1 h\left|V_{12}\right| 2 p 2 h\right\rangle}=98 \mathrm{keV}$, $\overline{\left\langle 2 p 2 h\left|V_{22}\right| 2 p 2 h\right\rangle}=104 \mathrm{keV}$, and $d_{2 p 2 h}=11.3 \mathrm{keV}$, which are evaluated for all the unperturbed $1 p 1 h$ and $2 p 2 h$ states in the energy interval of $E=20-30 \mathrm{MeV}$. Note also $d_{1 p 1 h}$ $\approx 0.5 \mathrm{MeV}$ for the $1 p 1 h$ states in the same interval. Very similar values are obtained for $\overline{\left\langle 1 p 1 h\left|V_{12}\right| 2 p 2 h\right\rangle}$ when we use the correlated $1 p 1 h$ states (TD states) instead of unperturbed $1 p 1 h$ 's.

It is also possible to evaluate $\gamma_{12}$ and $\gamma_{22}$ in a more direct way by using the strength functions of $1 p 1 h$ and $2 p 2 h$ states. Strength function of a $1 p 1 h$ state $|i, 1 p 1 h\rangle$ is given by

$$
S_{i, 1 p 1 h}(e)=\sum_{a}\langle i, 1 p 1 h \mid a\rangle^{2} \delta\left(e-E_{a}+E_{i, 1 p 1 h}\right)
$$

where $|a\rangle$ and $E_{a}$ are the eigenstates of the full calculation and their energies, respectively. $e$ denotes the relative energy from the energy centroid $E_{i, 1 p 1 h}$ of the $1 p 1 h$ state considered. Evaluating an average of $S_{i, 1 p 1 h}(e)$ over all the $1 p 1 h$ states, the average strength function of $1 p 1 h$ states is obtained, as shown in Fig. 10(a). Its FWHM gives an evaluation of the average spreading width $\gamma_{12}$ of $1 p 1 h$ states. Similar method can be used to evaluate the spreading width $\gamma_{22}$ of the $2 p 2 h$ states [cf. Fig. 10(b)]. This direct method gives $\gamma_{12}^{\mathrm{dm}}=1.5 \mathrm{MeV}$ and $\gamma_{22}^{\mathrm{dm}}=5.2 \mathrm{MeV}$.

It is noted that the values of the spreading width $\gamma_{12}$ are very different between the golden rule and the direct method. To investigate the difference, we evaluated root mean square values of interaction matrix elements of $V_{12}$ for individual 
(a)

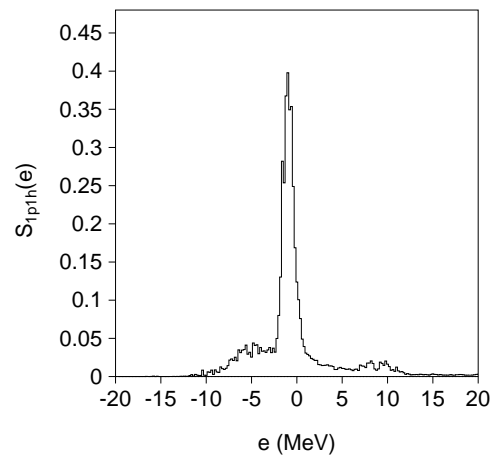

(b)

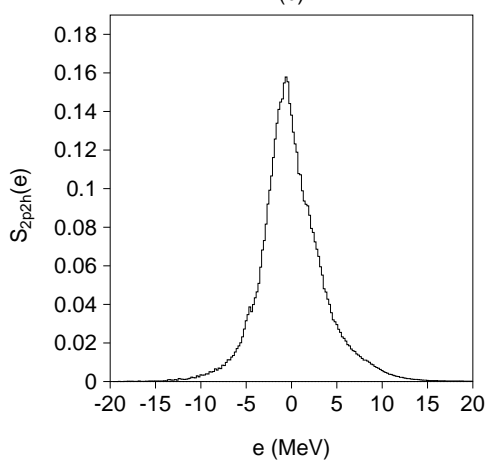

FIG. 10. (a) The strength function of the $1 p 1 h$ state averaged over all $1 p 1 h$ states within the energy interval $E=20-30 \mathrm{MeV}$ and (b) the strength function of the $2 p 2 h$ states averaged over the same energy interval.
$1 p 1 h$ states, and found that about one-third of the $1 p 1 h$ states have significantly large value $\simeq 120-200 \mathrm{keV}$ as compared with the other $1 p 1 h$ states which have small value $\simeq 30-70 \mathrm{keV}$. Corresponding golden rule estimates of the spreading width are $\gamma_{12}^{\mathrm{FG}}=8.3-22 \mathrm{MeV}$ and $\gamma_{12}^{\mathrm{FG}}$ $=0.5-2.8 \mathrm{MeV}$, respectively. The FWHM of the average strength function of $1 p 1 h$ states used in the direct method is dominated by the $1 p 1 h$ states having small FWHM. Accordingly, the direct method gives much smaller value $\left(\gamma_{12}^{\mathrm{dm}}\right.$ $=1.5 \mathrm{MeV})$ than that of the golden rule $\left(\gamma_{12}^{\mathrm{FG}}=5.3 \mathrm{MeV}\right)$.

\section{Dependence of LSD on $V_{12}$ and $V_{22}$}

In order to identify the origin of the observed energy scale $\epsilon_{\mathrm{f}}=1.7 \mathrm{MeV}$, we shall analyze the dependence of the fine structures of the strength functions on $V_{12}$ and $V_{22}$, which are intimately related to the spreading widths $\gamma_{12}$ and $\gamma_{22}$. For this analysis we rescale the interaction strength $V_{12}$ and $V_{22}$ by factors $\lambda_{12}$ and $\lambda_{22}$, respectively, and look into the local scaling dimension to see how the fine structures change.

It is seen from Fig. 11 that the fine structures probed by the local scaling dimension $D_{m}(\epsilon)$ strongly depend on $\lambda_{12}$. When the value of $\lambda_{12}$ is small, $\lambda_{12}=0.1$, the LSD exhibits very strong deviation from the GOE limit in almost all energy scales except at very small energy scale $\epsilon / d \sim 1$. As the value of $\lambda_{12}$ increases, the deviation becomes smaller and the LSD at small energy scales coincides with the GOE limit. The energy scale $\epsilon^{*}$ where the LSD start to deviate from the GOE limit moves to larger values as $\lambda_{12}$ increases. If $\lambda_{12}$ is increased more than the original value (e.g., $\lambda_{12}=1.7$ ), the LSD almost reaches the GOE limit over almost whole energy scales. The above dependence on $\lambda_{12}$ agrees very well with a similar behavior found in the simple doorway damping model [14]. (In that schematic model one can account for the spreading width $\gamma_{\mathrm{dw}}$ of the doorway states analytically.) We found in the schematic model that the energy scale $\epsilon^{*}$ is proportional to $\gamma_{\mathrm{dw}}$ as $\epsilon^{*} \approx \gamma_{\mathrm{dw}} / 5$ and the energy scale $\epsilon^{* *}$ where the LSD decreases most steeply corresponds to $\gamma_{\mathrm{dw}} \cdot \epsilon^{*}$ and $\epsilon^{* *}$ moves with the interaction $v$ between the doorway states and the background states since $\gamma_{\mathrm{dw}} \propto v^{2}$. This behavior is consistent with the $\lambda_{12}$ dependence in the present model. For quantitative evaluation, we compare the spreading width $\gamma_{12}$ of the $1 p 1 h$ TD states, which are considered here as the doorway states, and the energy scale $\epsilon_{\mathrm{f}}$ where the deviation of the LSD from the GOE limit is maximum. Here we adopt the energy scale $\epsilon_{\mathrm{f}}$ in place of $\epsilon^{* *}$, whose extraction is not easy in the present model due to fluctuation in the LSD. The energy scale $\epsilon_{\mathrm{f}}=1.7 \mathrm{MeV}$ found for the original interaction (i.e., $\lambda_{12}=1$ ) agrees with the spreading width $\gamma_{12}$ of the $1 p 1 h$ states if we adopt the value $\gamma_{12}^{\mathrm{dm}}=1.5 \mathrm{MeV}$ obtained by the direct evaluation. The agreement is a little worse if we refer to the value $\gamma_{12}^{\mathrm{FG}}=5.3 \mathrm{MeV}$ derived from the golden rule estimate. We expect that the fine structures are dominated by the group of $1 p 1 h$ states having smaller spreading width $\gamma_{12} \sim 1 \mathrm{MeV}$, while the remaining $1 p 1 h$ states with $\gamma_{12}>10 \mathrm{MeV}$, which is larger than the total width of the giant resonance, do not contribute to the fine structure. The comparison with the spreading width $\gamma_{12}^{\mathrm{dm}}$ by the direct evaluation would be more appropriate since $\gamma_{12}^{\mathrm{dm}}$ reflects mainly the first group. (On the other hand, $\gamma_{12}^{\mathrm{FG}}$ based on the root mean square of interaction matrix elements puts emphasis on the other group. See the preceding section.) The $\lambda_{12}$ dependence and the comparison with $\gamma_{12}$ indicate an approximate relation $\epsilon_{\mathrm{f}} \sim \gamma_{12}$, for which we estimate an ambiguity by a factor of 2 .

Figure 12 shows $\lambda_{22}$ dependence of the LSD. We immediately see that dependence on $\lambda_{22}$ is much weaker than that on $\lambda_{12}$. For $\lambda_{22}<0.2$, the LSD exhibits deviation from the GOE limit for rather wide interval of energy scale, but the deviation itself is not very large. As $\lambda_{22}$ increases, the LSD approaches the GOE limit at small energy scales. (At $\lambda_{22}$ $=0.5$, for instance, the local scaling dimensions almost follow the GOE when $\epsilon \lesssim 10 d$.) For $\lambda_{22}>0.5$ the LSD is not sensitive to the change in $\lambda_{22}$, and there remains the same deviation from the GOE limit even up to the maximal choice of $\lambda_{22}=2.0$.

The insensitivity to $\lambda_{22}$ for $\lambda_{22}>0.5$ may be understood as follows: We first note that the energy scale of the mixing among $2 p 2 h$ states is given by the spreading width $\gamma_{22}$ of the $2 p 2 h$ states, and that the mixing properties are expected to show up in the GOE behaviors for small energy scales satisfying $\epsilon<\gamma_{22}$. In the case of the original strength $\lambda_{12}=\lambda_{22}$ $=1$, the spreading width $\gamma_{22}^{\mathrm{dm}}=5.2 \mathrm{MeV}$ of the $2 p 2 h$ states is larger than the spreading width $\gamma_{12}^{\mathrm{dm}}=1.5 \mathrm{MeV}$ of the $1 p 1 h$ TD states. If $\lambda_{22}<0.5$, the opposite relation $\gamma_{22}<\gamma_{12}$ is realized (n.b. $\gamma_{22} \propto \lambda_{22}^{2}$ and $\gamma_{12} \propto \lambda_{12}^{2}$ ), and hence the energy scale where the deviation from the GOE limit starts is determined by $\gamma_{22}$. In the case of $\lambda_{22}>0.5$ (including the case of the original strength), on the other hand, we have $\gamma_{12}<\gamma_{22}$, and the deviation from the GOE limit is governed by $\gamma_{12}$. In principle, we can consider a possibility that the LSD exhibits 

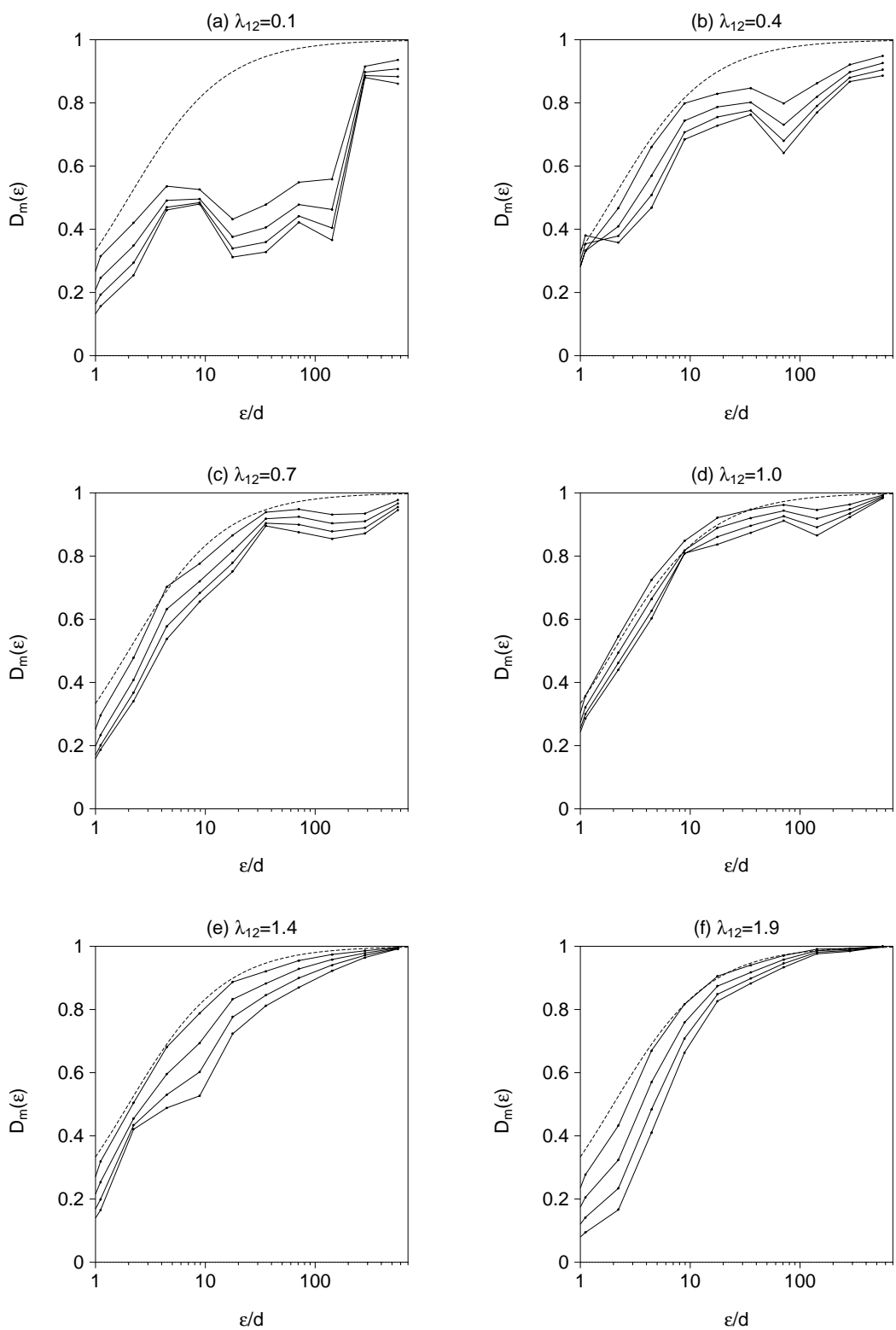

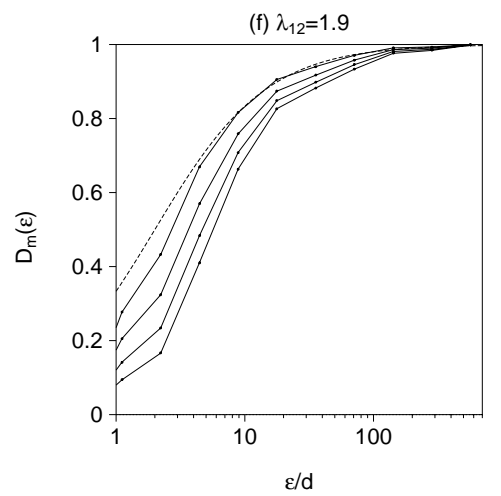

FIG. 11. The local scaling dimensions $D_{m}(\epsilon)$ $(m=2-5)$ for the ISGQR calculated by changing values of $\lambda_{12}$, which is chosen as (a) $\lambda_{12}=0.1$, (b) $\lambda_{12}=0.4$, (c) $\lambda_{12}=0.7$, (d) $\lambda_{12}=1.0$, (e) $\lambda_{12}=1.4$, and (f) $\lambda_{12}=1.9$. Here $\lambda_{12}$ represents the ratio between the adopted values of the Hamiltonian matrix elements between the TD $1 p 1 h$ states and $2 p 2 h$ states, and those of original values. The dashed curve represents $D_{2}(\epsilon)$ for the GOE. the two energy scales $\gamma_{12}$ and $\gamma_{22}$ separately. However, the deviation related to $\gamma_{22}$ is small even when $\gamma_{22}<\gamma_{12}$ $\left(\lambda_{22}<0.5\right)$, making it difficult to extract the energy scale associated with $\gamma_{22}$. In the case of the original interaction strength, the spreading widths $\gamma_{22}^{\mathrm{dm}}=5.2 \mathrm{MeV}$ is close to the total width $\gamma_{G R}(\sim 5 \mathrm{MeV})$ of the giant resonance, and thus it is difficult to be probed. (Notice that we adopted a smoothing width of $5 \mathrm{MeV}$.)

Summarizing the above two analyses, the characteristic energy scale of the fine structures is related to the spreading width $\gamma_{12}$ of the $1 p 1 h$ TD states, which are considered as the doorway states in the giant resonance decay.

\section{Isovector strength function}

We have also analyzed the strength distribution for the isovector quadrupole operator. Figures 13(a) and 13(b) show the partition function and the local scaling dimension, respectively, for the isovector strength function in the energy interval $E=20-30 \mathrm{MeV}$. The behavior of the LSD is similar to that of isoscalar strength function. Namely, the local scaling dimension almost follows the GOE limit at smaller energy scales whereas the deviation from the GOE limit is recognized at intermediate energy scales. We also verified that the change of the value of $\epsilon_{\mathrm{f}}$ is quite sensitive to the change of $\lambda_{12}$ compared to the change of $\lambda_{22}$ as in the case of the ISGQR. Characteristic energy scale of the deviation reads $\epsilon_{\mathrm{f}} \sim 70 d \sim 0.9 \mathrm{MeV}$. This value is smaller than that for the isoscalar strengths $\left(\epsilon_{\mathrm{f}} \sim 1.7 \mathrm{MeV}\right)$ by about a factor of 2 . We have investigated the rms value of the interaction matrix elements to see the isospin dependence of the spreading width of the Tamm-Dancoff states, but we could not find clear difference between the IS and IV modes.

\section{CONCLUSIONS}

We have analyzed fine structures of the giant quadrupole resonances described by means of the microscopic shell 

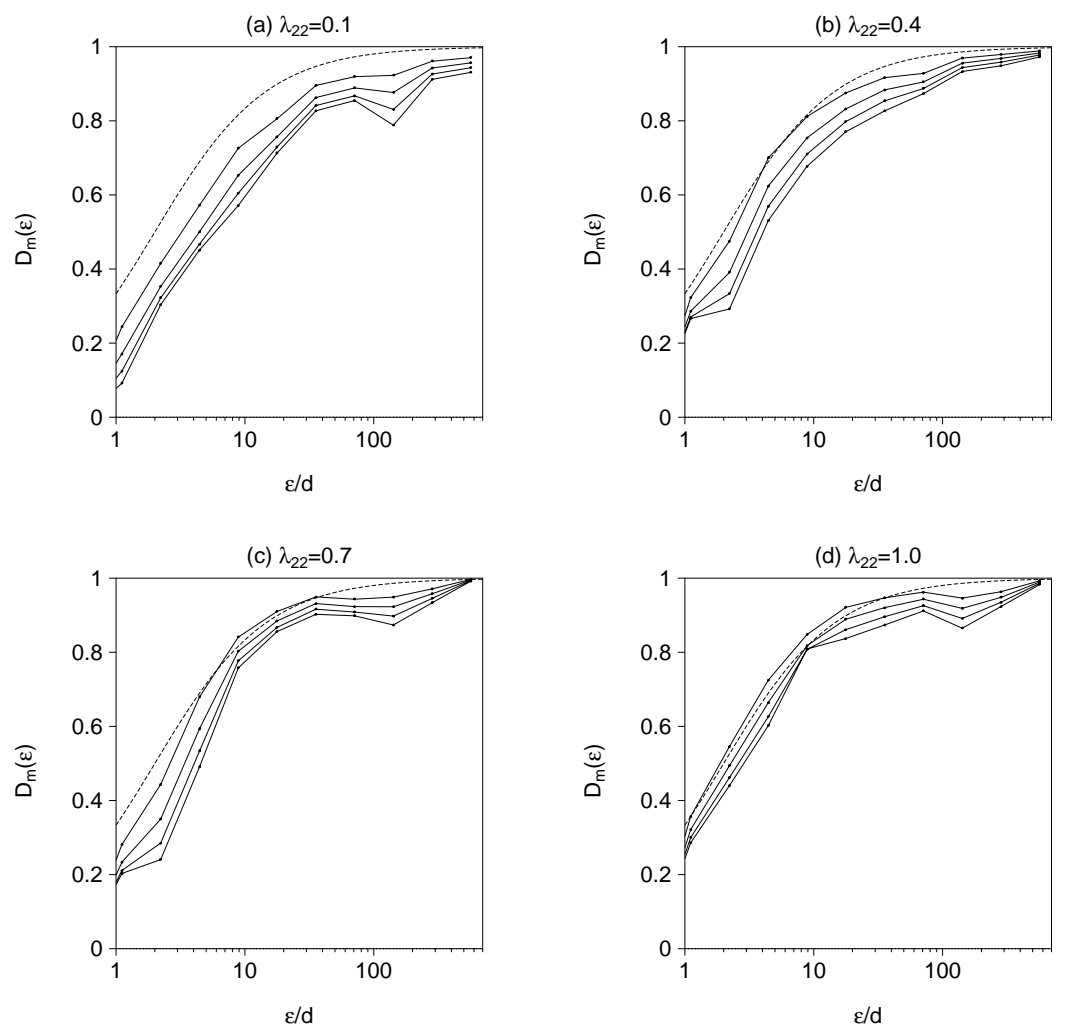

FIG. 12. The local scaling dimensions $D_{m}(\epsilon)(m=2-5)$ for the ISGQR calculated by changing values of $\lambda_{22}$, which is chosen as (a) $\lambda_{22}=0.1$, (b) $\lambda_{22}=0.4$, (c) $\lambda_{22}$ $=0.7$, (d) $\lambda_{22}=1.0$, (e) $\lambda_{22}=1.6$, and (f) $\lambda_{22}=1.9$. Here $\lambda_{22}$ represents the ratio between the adopted values of the Hamiltonian off-diagonal matrix elements between $2 p 2 h$ states and those of original values. The dashed curve represents $D_{2}(\epsilon)$ for the GOE.
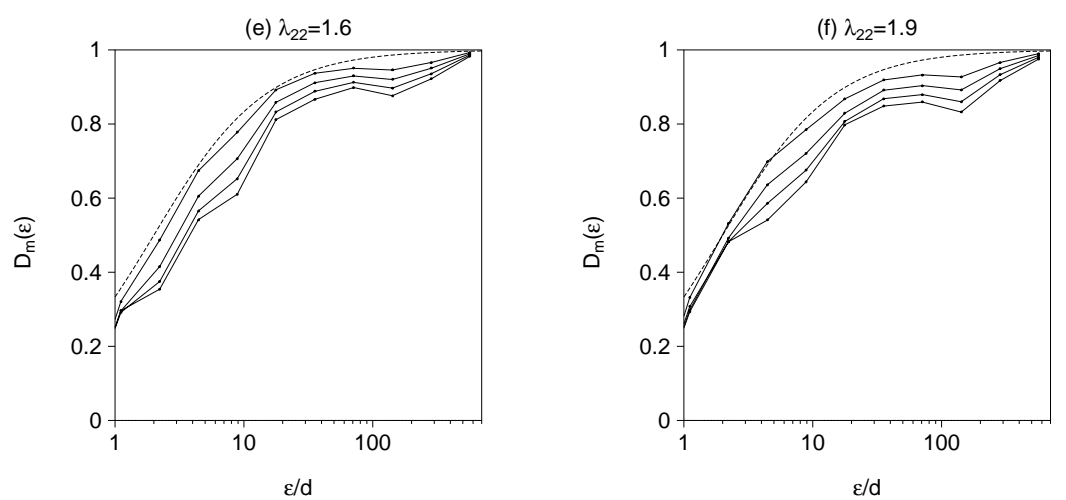

model including up to $2 p 2 h$ configurations. By applying the scaling analysis of strength fluctuations that utilizes the local scaling dimension, we extracted the energy scale $\epsilon_{\mathrm{f}}$ $=1.7 \mathrm{MeV}$ for the ISGQR and $\epsilon_{\mathrm{f}}=0.9 \mathrm{MeV}$ for the IVGQR, which characterize the fine structure. We discussed the origin

of the characteristic energy scale in terms of the spreading widths of the $1 p 1 h$ and $2 p 2 h$ states. We found a clear correlation between the characteristic energy scale and the spreading width $\gamma_{12}$ of the $1 p 1 h$ Tamm-Dancoff states, which play a role of doorway states in the present shell model descrip-
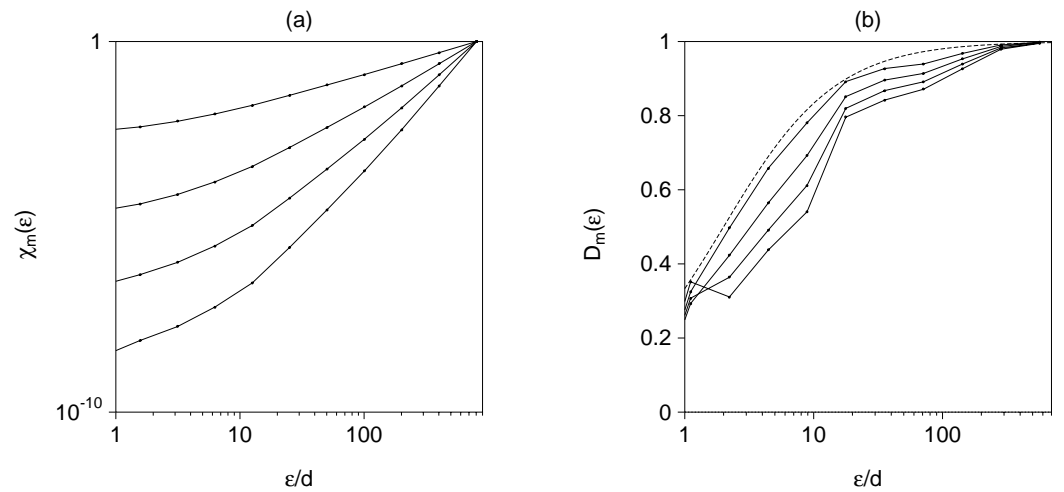

FIG. 13. (a) The partition function $\chi_{m}(\boldsymbol{\epsilon})(m=2-5)$ for the IVGQR corresponding to the normalized strength function plotted in Fig. 6(b). (b) Its local scaling dimension $D_{m}(\epsilon)$ for $m=2-5$. The dashed curve represents $D_{2}(\epsilon)$ for the GOE. 
tion of the giant quadrupole resonances in ${ }^{40} \mathrm{Ca}$.

In the present paper, we intended to illustrate how the scaling analysis can be utilized to investigate the fine structures and their origin. Although the adopted model has some realistic features, we do not claim that the present shell model predicts all the quantitative features of the quadrupole strength distributions since the particle escaping width, for instance, is neglected in the present model. Also, other mechanisms such as the surface phonon coupling are not explicitly taken into account in the present model. Apparently, one may need a more realistic model if one intends to make a quantitative prediction or comparison with the experiment. Instead, we would like to emphasize that the local scaling analysis provides a general tool for quantitative investigation of the fine structures, and the scaling analysis itself can be applied to any kinds of models which exhibit fine structures in the strength functions.

\section{ACKNOWLEDGMENTS}

The authors acknowledge helpful comments by K. Matsuyanagi. The numerical calculations were performed at Yukawa Institute Computer Facility and on SX-5 at RCNP, Osaka University. This research was partly supported by Grant-in-Aid for Science Research from the Japan Society for the Promotion of Science (No. 14540272).
[1] G. F. Bertsch, P. F. Bortignon, and R. A. Broglia, Rev. Mod. Phys. 55, 287 (1983).

[2] Electric and Magnetic Giant Resonances in Nuclei, edited by J. Speth (World Scientific, Singapore, 1991).

[3] M. N. Harakeh and A. van der Woude, Giant Resonances (Oxford University Press, Oxford, 2001).

[4] F. J. Dyson, J. Math. Phys. 3, 140 (1962); 3, 157 (1962); 3, 166 (1962).

[5] M. L. Mehta, Random Matrices, 2nd ed. (Academic, New York, 1991).

[6] O. Bohigas and M.-J. Giannoni, Lecture Notes in Physics (Springer, New York, 1984), Vol. 219, p. 1.

[7] T. A. Brody, J. Flores, J. B. French, P. A. Mello, A. Pandy, and S. S. M. Wong, Rev. Mod. Phys. 53, 385 (1981).

[8] A. Bohr and B. R. Mottelson, Nuclear Structure (Benjamin, New York, 1996), Vol. 1, Chap. 2.

[9] R. V. Haq, A. Pandey, and O. Bohigas, Phys. Rev. Lett. 48, 1086 (1982).

[10] P. G. Hansen, B. Jonson, and A. Richter, Nucl. Phys. A518, 13 (1990).

[11] J. C. Hardy, B. Jonson, and P. G. Hansen, Nucl. Phys. A305, 15 (1978).

[12] G. Kilgus, G. Kühner, S. Müller, A. Richter, and W. Knüpfer,
Z. Phys. A 326, 41 (1987).

[13] S. Kamerdzhiev, J. Lisantti, P. von Neumann-Cosel, A. Richter, G. Tertychny, and J. Wambach, Phys. Rev. C 55, 2101 (1997).

[14] H. Aiba and M. Matsuo, Phys. Rev. C 60, 034307 (1999).

[15] D. Lacroix and Ph. Chomaz, Phys. Rev. C 60, 064307 (1999).

[16] D. Lacroix, A. Mai, P. von Neumann-Cosel, A. Richter, and J. Wambach, Phys. Lett. B 479, 15 (2000).

[17] A. Bohr and B. R. Mottelson, Nuclear Structure (Benjamin, New York, 1996), Vol. 1, Chap. 2D.

[18] H. G. E. Hentschel and I. Procaccia, Physica D 8, 435 (1983).

[19] T. C. Halsey, M. H. Jensen, L. P. Kadanoff, I. Procaccia, and B. I. Shraiman, Phys. Rev. A 33, 1141 (1986).

[20] B. Schwesinger and J. Wambach, Nucl. Phys. A426, 253 (1984).

[21] S. Drożdż, S. Nishizaki, and J. Wambach, Phys. Rev. Lett. 72, 2839 (1994).

[22] S. Drożdż, S. Nishizaki, J. Wambach, and J. Speth, Phys. Rev. Lett. 74, 1075 (1995).

[23] P. Ring and P. Schuck, The Nuclear Many-Body Problem (Springer, New York, 1980), Chap. 2.9.

[24] S. Drożdż, S. Nishizaki, J. Speth, and J. Wambach, Phys. Rev. C 49, 867 (1994). 